www.nature.com/jhg

\title{
SNPs in axon guidance pathway genes and susceptibility for Parkinson's disease in the Korean population
}

\author{
Jong-Min Kim ${ }^{1}$, Sue K Park ${ }^{2,3,4}$, Jae Jeong Yang, ${ }^{2,3}$, Eun-Soon Shin ${ }^{5}$, Jee-Young Lee ${ }^{1}$, Ji Young Yun ${ }^{1}$, \\ Ji Seon Kim ${ }^{1}$, Sung Sup Park ${ }^{6}$ and Beom S Jeon ${ }^{1}$
}

Single-nucleotide polymorphisms (SNPs) in genes of the axon guidance pathway have been reported to be a possible susceptibility factor for Parkinson's disease (PD). This study investigated whether the genetic variability in the axon guidance pathway is a susceptibility factor in PD patients in the Korean population. A total of 373 patients and 384 healthy subjects were included. A set of 22 SNPs was analyzed, and the risk of PD was evaluated using odds ratios in an unconditional and conditional logistic regression models of age- and gender-matched subsets. A multidimensionality reduction (MDR) analysis was performed to explore potential gene-gene interactions. SNPs in the DCC, CHP, RRAS2 and EPHB1 genes of the axon guidance pathway showed significant associations with PD. The DCC rs17468382 and EPHB1 rs2030737 SNPs may be associated with increased PD risk, and the CHP rs6492998 and RRAS2 rs2970332 SNPs may be associated with reduced PD risk. However, no significant interactions for PD risk were found in the MDR analysis and logistic regression analysis using SNP interaction terms. This study supports that only four of the selected 22 SNPs are regulating factors associated with PD in the Korean population. However, no interactions were found among the SNPs, suggesting that the effect for the pathway as a whole is not greater than that for single genes in the Korean population. Further investigations involving populations of various ethnicities and other genetic markers and models are warranted.

Journal of Human Genetics (2011) 56, 125-129; doi:10.1038/jhg.2010.130; published online 18 November 2010

Keywords: axon guidance pathway; Parkinson's disease; single-nucleotide polymorphisms

\section{INTRODUCTION}

Parkinson's disease (PD) is a common neurodegenerative disease, most cases of which are sporadic. ${ }^{1}$ Several mutations have been identified as being associated with the Mendelian forms of PD., ${ }^{2,3}$ However, much work remains to be performed before the genetic causes of sporadic PD can be identified. Genome-wide association studies have revealed several putative markers for $\mathrm{PD}^{4-9}$ Semaphorin proteins have been shown to have a role in the axon guidance of developing dopaminergic neurons and to interact with other proteins participating in guiding axonal development. ${ }^{10-12}$ These experimental findings and the data from genetic studies led Lesnick et al..$^{13}$ to investigate the combined actions of multiple genes of the axon guidance pathway predisposing to $\mathrm{PD}$.

Lesnick et al. ${ }^{13}$ reported that multiple single-nucleotide polymorphisms (SNPs) in axon guidance pathway genes were significant predictors of PD susceptibility. Constructed sets of SNPs in axon guidance pathway genes were subsequently found highly predictive of PD susceptibility, survival time free of PD and age at onset of PD.

This genomic pathway approach to an association between PD and the axon guidance pathway has generated considerable scientific interest. Srinivasan et al. ${ }^{14}$ developed a statistical method for finding pathways associated with PD that controls for pathway size. They confirmed the axon guidance association reported by Lesnick et al., ${ }^{13}$ and also indicated that variation in the ubiquitin-mediated proteolysis and T-cell receptor signaling pathways may have been predictive of PD susceptibility in their patients. However, they found that applying their method to the data set of the genome-wide association study of Fung et al. ${ }^{5}$ weakened the association of the axon guidance pathway with PD.

The above discrepancy regarding the contribution of the axon guidance pathway to PD between data sets of individual genetic studies is further complicated by another study. ${ }^{15} \mathrm{Li}$ et al. tested the

\footnotetext{
${ }^{1}$ Department of Neurology, Seoul National University College of Medicine, MRC and BK-21, Parkinson Study Group, Clinical Research Institute, Seoul National University Hospital and Bundang Hospital, Seoul, Korea; ${ }^{2}$ Department of Preventive Medicine, Seoul National University College of Medicine, MRC and BK-21, Seoul, Korea; ${ }^{3}$ Cancer Research Institute, Seoul National University, Seoul, Korea; ${ }^{4}$ Institute of Health Management and Policy, Seoul National University, Seoul, Korea; ${ }^{5}$ NA Link Bioinformatics 1 Team, Seoul, Korea and ${ }^{6}$ Department of Laboratory Medicine, Seoul National University College of Medicine, MRC and BK-21, Clinical Research Institute, Seoul National University Hospital, Seoul, Korea

Correspondence: Professor BS Jeon, Department of Neurology, Seoul National University Hospital, Chongno-Ku Yunkeun-Dong 28, Seoul 110-744, Korea.

E-mail: brain@snu.ac.kr
}

Received 18 June 2010; revised 10 September 2010; accepted 24 September 2010; published online 18 November 2010 
risk panel of 23 SNPs proposed by Lesnick et al. ${ }^{13}$ in two PD sample sets. ${ }^{15}$ They reported that only one SNP was significantly associated with PD susceptibility in one of the sample sets. Their multimarker analysis found only a weak association between SNP and PD susceptibility in one sample set $(\mathrm{OR}=1.07, P=0.049)$, with no significance in the other sample set $(\mathrm{OR}=1, P=0.98)$. On the basis of these results, the authors doubted that the axon guidance pathway has a role in PD genetics.

Data on the association between the axon guidance pathway and $\mathrm{PD}$, therefore, appears to be inconsistent across studies, and moreover the analyzed populations have been limited to those with Caucasian ethnicity. We previously showed that the observed genetic risk may differ significantly among different ethnic groups with different genetic compositions. ${ }^{16}$ Therefore, in this study, we investigated whether the genetic variability in the axon guidance pathway is a susceptibility factor for PD in the Korean population.

\section{MATERIALS AND METHODS}

\section{Subjects}

Gene samples were obtained from the gene bank at the Movement Disorder Division of Seoul National University Hospital. All patients and controls were native Koreans. The institutional review board of Seoul National University Hospital approved the study. Blood samples were collected after written informed consent was obtained from each participant. PD was diagnosed according to the United Kingdom Parkinson Disease Society Brain Bank criteria, with the exception of the positive family history criterion. ${ }^{17}$ The patients were screened for LRRK2 G2019S, SCA2, SCA17 and SNCA mutations, and for Parkin, PINK1 and DJ-1 mutations in patients whose age at onset was $\leqslant 40$ years, and those who were positive were excluded from the study. ${ }^{18-22}$

A total of $373 \mathrm{PD}$ patients were included in the study. DNA was also obtained from 384 healthy subjects with no family history of parkinsonism from the gene database at the Department of Laboratory Medicine, Seoul National University Hospital. These normal controls were either healthy spouses of PD patients or those who presented for routine health examinations.

\section{Genetic analysis}

This study analyzed 23 SNPs that were suggested as being predictive of PD susceptibility by Lesnick et al. ${ }^{13}$ Those SNPs with a minor allele frequency of $>1 \%$ were genotyped in all of the samples. Venous blood samples were drawn and genomic DNA was extracted using standard techniques. The genotypes of 16 SNPs were screened with a single base primer extension assay using the ABI PRISM SNaPShot Multiplex kit (Applied Biosystems, Foster City, CA, USA) according to the manufacturer's recommendations. TaqMan analysis was applied to seven SNPs. Analyses were performed using GeneMapper software (version 4.0, Applied Biosystems, Foster City, CA, USA). Details of the genotyping and the primer sets used for the assay are summarized in Supplementary Table S3. The accuracy of genotyping was ensured by performing blind tests on duplicate samples, and including negative controls.

\section{Statistical analysis}

One of the 23 SNPs, rs13386128, was excluded from the final analysis because of having a minor allele frequency of $<0.01$. Additionally, seven patients and five normal controls were excluded because of low genotyping rate $(<90 \%)$. We finally analyzed 22 SNPs among 745 subjects (366 cases and 379 controls), with a genotyping rate of $95.8 \%$.

The basic characteristics of all the subjects were compared by applying the Pearson's $\chi^{2}$-test or Student's $t$-test, depending on the variable type (that is, continuous or discrete). Hardy-Weinberg equilibrium was evaluated in the control group using the $\chi^{2}$-test or Fisher's exact test. We excluded SNPs with an Hardy-Weinberg equilibrium cutoff of $<0.05$ in the analyses. The association between SNPs and PD was analyzed in additive models, using a code of 0 for homozygous genotypes with two major alleles, 1 for heterozygous genotypes with a major and a minor allele, and 2 for homozygous genotypes with two minor alleles.
The permutated $P$-values in single SNP analysis were determined from 10000 permutation tests. To avoid spurious associations with false-positive outcomes, the false discovery rate was also estimated using the BenjaminiHochberg method. ${ }^{23}$

The PD risks were quantified using two ORs and a hazard ratio. The first ORs and $95 \%$ confidence intervals (CIs) were computed using an unconditional logistic regression model whilst adjusting for age and gender in all of the subjects. In complete data sets, we matched age and gender between patients and controls, and the second ORs were computed using a conditional logistic regression model for age- and gender-matched subsets. Moreover, to investigate the association between the SNPs and the survival time free of PD, we estimated hazard ratios and 95\% CIs using the Cox proportional-hazards regression model and survival rates using the log-rank test. The follow-up time was considered to be the age at the diagnosis of PD for the patients, and the age at the enrollment for the control subjects.

The genetic risk score was determined from the number of risk alleles and risk estimates for rare alleles. ${ }^{24}$ It was calculated by multiplying risk estimates ( $\beta$-values calculated in the additive model) for each SNP by the number of risk alleles carried by each person ( 0 for homozygous genotypes with two major alleles, 1 for heterozygous genotypes with a major and a minor allele, and 2 for homozygous genotypes with two minor alleles). ${ }^{24}$

We explored the potential gene-gene interactions between the 22 genes of the axon guidance pathway by performing a multidimensionality reduction (MDR) analysis, which is a non-parametric data-mining approach for detecting a potential gene-gene or gene-environment interaction, and providing selective models of high-order gene combinations. ${ }^{25,26}$ A naïve Bayes classifier in the context of a 10-fold cross-validation was used to estimate the test accuracy of the best one-, two-, three- and four-factor models. In a sign test the cutoff for statistical significance was set at the 0.05 level. To assess the association between genotype combinations suggested by MDR methods, MDR ORs and $95 \%$ CIs were computed using both unconditional and conditional logistic regression models. Finally, we conducted logistic regression analysis focusing on the interaction suggested by Lesnick et al. ${ }^{13}$ by using the interaction terms of SNPs.

All statistical analyses were performed using SAS software version 9.1 (SAS Institute, Cary, NC, USA), PLINK software version 1.06 (available at http:// pngu.mgh.harvard.edu/purcell/plink) ${ }^{27}$ and MDR software version 11.0 (available at http://www.epistasis.org) (Supplementary Text S1-S5).

\section{RESULTS}

The demographic characteristics of the subjects are listed in Table 1 and Supplementary Table S1. Among all 757 subjects, the gender distribution did not differ significantly between patients and normal controls, but the mean age was significantly lower for patients than for controls. To avoid possible effects of age and gender on the PD risk, we generated 290 age- and gender-matched sets for subgroup analysis (Table 1). An analysis of all of the subjects produced similar results (data not shown).

The results of the genotyping of the 22 SNPs are presented in Table 2 and Supplementary Table S2. All allele distributions of the patients and normal controls were concordant with Hardy-Weinberg equilibrium $(>0.05)$. Of the $22 \mathrm{SNPs}$ in axon guidance pathway genes, 2 SNPs (rs17468382 in DCC and rs6492998 in CHP) were significantly associated with $\mathrm{PD}$ (raw and permutated $P<0.05$ ). SEMA5A was not associated with PD risk. For carriers of the SNPs in the DCC, CHP, RRAS2, and EPHB1 genes, the ORs were 2.03, 0.77, 0.81 , and 1.29 , respectively $(P<0.10)$. The frequencies of the $D C C$ rs17468382 and EPHB1 rs2030737 variants were higher in PD patients than in normal controls, which means that these SNPs are potential risk factors for PD. The frequencies of the CHP rs6492998 and RRAS2 rs2970332 SNPs were lower in PD patients than in normal controls, suggesting that these SNPs exert protective effects. However, under the multiple comparison tests based on the false discovery rate, the significance of these four SNPs disappeared (false discovery rate 
Table 1 Basic characteristics of study subjects

\begin{tabular}{|c|c|c|c|c|c|c|}
\hline & \multicolumn{3}{|c|}{ Total subjects } & \multicolumn{3}{|c|}{ Matched subjects } \\
\hline & PD patients $(\mathrm{N}=373)^{\mathrm{b}}$ & Normal controls $(\mathrm{N}=384)$ & $P$ & $P D$ patients $(\mathrm{N}=290)^{\mathrm{b}}$ & Normal controls $(\mathrm{N}=290)$ & $P$ \\
\hline \multicolumn{7}{|l|}{ Gender } \\
\hline Female (\%) & $202(54.2)$ & $217(56.5)$ & 0.5147 & $173(59.7)$ & $173(59.7)$ & 1.0000 \\
\hline \multicolumn{7}{|l|}{ Age } \\
\hline Mean (s.d.) & $62.8(9.1)$ & $64.5(9.1)$ & 0.0127 & $63.9(8.5)$ & $63.9(8.5)$ & 0.9530 \\
\hline \multicolumn{7}{|l|}{ Age of onset } \\
\hline Mean (s.d.) & $53.3(9.4)$ & - & NA & $53.6(8.8)$ & - & NA \\
\hline
\end{tabular}

Abbreviations: NA, not available; PD, Parkinson's disease.

${ }^{a}$ Among total study subjects, one versus one matching by gender and age ( \pm 1 year).

bCases diagnosed as having PD.

Table 2 Single marker association with Parkinson's disease susceptibility

\begin{tabular}{|c|c|c|c|c|c|c|c|c|c|c|c|}
\hline Gene & $d b S N P I D$ & $\begin{array}{c}\text { Raw } \\
\text { P-value a }\end{array}$ & $\begin{array}{c}\text { Permutated } \\
\text { P-value }\end{array}$ & Allele & $\begin{array}{l}\text { MAF } \\
(\%)^{\mathrm{c}}\end{array}$ & $\begin{array}{c}\text { Chromosome } \\
\text { no. }\end{array}$ & $\begin{array}{c}\text { Chromosome } \\
\text { position }\end{array}$ & $F D R B H^{\mathrm{d}}$ & OR $(95 \% C l)^{\mathrm{e}}$ & $H R(95 \% C l)^{\mathrm{e}}$ & $\begin{array}{c}\text { Conditional } \\
\text { OR }(95 \% \mathrm{Cl})^{\mathrm{f}}\end{array}$ \\
\hline$D C C$ & rs17468382 & $0.02168^{*}$ & $0.02652^{*}$ & $\mathrm{C} / \mathrm{T}$ & $C(1.98)$ & 18 & 48515009 & 0.3107 & $2.03(1.06-3.86)^{*}$ & $1.80(1.23-2.64)^{*}$ & $1.92(1.00-3.85)^{* *}$ \\
\hline $\mathrm{CHP}$ & rs6492998 & $0.02825^{*}$ & $0.0292^{*}$ & $\mathrm{G} / \mathrm{A}$ & G (27.7) & 15 & 39333923 & 0.3107 & $0.77(0.60-0.97)^{*}$ & $0.84(0.70-1.00)^{*}$ & $0.77(0.59-1.01)^{* *}$ \\
\hline RRAS2 & rs2970332 & 0.05193 & 0.05177 & $\mathrm{C} / \mathrm{T}$ & C (45.9) & 11 & 14317011 & 0.3808 & $0.81(0.66-0.99)^{*}$ & $0.86(0.74-0.99)^{*}$ & $0.79(0.62-0.99)^{*}$ \\
\hline$E P H B 1$ & rs2030737 & 0.08156 & 0.08095 & $\mathrm{~T} / \mathrm{C}$ & $\mathrm{T}(13.6)$ & 3 & 136292067 & 0.4486 & $1.29(1.00-1.71)^{* *}$ & $1.17(0.97-1.42)$ & $1.26(0.91-1.75)$ \\
\hline SLIT3 & rs9688032 & 0.1915 & 0.1925 & $\mathrm{~A} / \mathrm{T}$ & $A(51.2)$ & 5 & 168488773 & 0.8427 & $0.86(0.70-1.06)$ & $0.91(0.79-1.05)$ & 0.87 (0.69-1.09) \\
\hline$P L X N C 1$ & rs2068435 & 0.2389 & 0.2438 & $\mathrm{C} / \mathrm{T}$ & C (19.0) & 12 & 93185105 & 0.8758 & $0.85(0.65-1.12)$ & $0.89(0.73-1.09)$ & $0.87(0.64-1.17)$ \\
\hline SEMA5A & rs12658266 & 0.3814 & 0.3855 & $\mathrm{G} / \mathrm{A}$ & G (26.9) & 5 & 9358725 & 0.9851 & 1.09 (0.87-1.37) & $1.00(0.85-1.17)$ & $1.12(0.87-1.44)$ \\
\hline RAC2 & rs739043 & 0.4766 & 0.5058 & $\mathrm{C} / \mathrm{T}$ & C (36.1) & 22 & 35975176 & 0.9851 & $0.92(0.74-1.15)$ & $0.91(0.78-1.07)$ & $0.85(0.66-1.11)$ \\
\hline UNC5C & rs4444836 & 0.5464 & 0.5462 & $\mathrm{C} / \mathrm{T}$ & C (37.3) & 4 & 96607674 & 0.9851 & $0.92(0.74-1.14)$ & $0.97(0.83-1.14)$ & $0.96(0.75-1.22)$ \\
\hline NTNG1 & rs11185076 & 0.5658 & 0.5577 & $\mathrm{~T} / \mathrm{A}$ & $\mathrm{T}(48.9)$ & 1 & 107598223 & 0.9851 & $1.06(0.86-1.31)$ & $1.08(0.93-1.25)$ & $1.01(0.79-1.28)$ \\
\hline GSK3B & rs16830689 & 0.5890 & 0.5841 & $\mathrm{G} / \mathrm{C}$ & $\mathrm{G}(7.25)$ & 3 & 121253961 & 0.9851 & $0.89(0.59-1.34)$ & $0.91(0.68-1.22)$ & $0.91(0.57-1.48)$ \\
\hline UNC5C & rs11097458 & 0.5915 & 0.5944 & $A / G$ & $A(38.5)$ & 4 & 96318390 & 0.9851 & $0.94(0.77-1.15)$ & $0.93(0.81-1.08)$ & $0.95(0.75-1.19)$ \\
\hline EFNA5 & rs153690 & 0.7486 & 0.7427 & $C / G$ & C (39.1) & 5 & 106836832 & 0.9851 & $1.04(0.84-1.27)$ & $1.03(0.89-1.19)$ & $1.05(0.82-1.34)$ \\
\hline PAK4 & rs17641276 & 0.761 & 0.7755 & $\mathrm{G} / \mathrm{A}$ & G (40.4) & 19 & 44365874 & 0.9851 & $0.97(0.79-1.21)$ & $0.95(0.81-1.11)$ & $0.95(0.73-1.23)$ \\
\hline РPРЗСA & rs2044041 & 0.8006 & 0.8079 & $\mathrm{~T} / \mathrm{A}$ & $\mathrm{T}(14.7)$ & 4 & 102454392 & 0.9851 & $0.98(0.74-1.31)$ & $0.94(0.77-1.16)$ & $0.91(0.66-1.26)$ \\
\hline PLXNA2 & rs6656034 & 0.8039 & 0.8115 & $\mathrm{~T} / \mathrm{C}$ & $\mathrm{T}(44.9)$ & 1 & 206305799 & 0.9851 & $1.02(0.83-1.25)$ & $1.02(0.88-1.18)$ & $1.10(0.87-1.39)$ \\
\hline PAK 7 & rs2072952 & 0.8316 & 0.8431 & $A / G$ & A (37.9) & 20 & 9473601 & 0.9851 & $1.03(0.83-1.27)$ & $1.02(0.88-1.19)$ & $1.06(0.84-1.34)$ \\
\hline MRAS & rs4678260 & 0.8809 & 0.8914 & $\mathrm{C} / \mathrm{T}$ & C (24.9) & 3 & 139572203 & 0.9851 & $1.02(0.81-1.29)$ & $1.04(0.88-1.22)$ & $1.09(0.84-1.42)$ \\
\hline EPHB2 & rs10917325 & 0.906 & 0.9104 & $\mathrm{~T} / \mathrm{C}$ & $\mathrm{T}(31.9)$ & 1 & 23081865 & 0.9851 & $0.990 .80-1.24)$ & $1.01(0.87-1.19)$ & $0.91(0.70-1.17)$ \\
\hline$C D C 42$ & rs12740705 & 0.9136 & 0.9211 & $\mathrm{~T} / \mathrm{C}$ & $\mathrm{T}(13.0)$ & 1 & 22293700 & 0.9851 & $1.02(0.75-1.38)$ & $0.92(0.75-1.14)$ & $0.83(0.58-1.19)$ \\
\hline GNAI3 & rs6692804 & 0.9403 & 0.9355 & $A / G$ & $A(28.1)$ & 1 & 109910265 & 0.9851 & $1.01(0.81-1.26)$ & $1.03(0.88-1.20)$ & $1.01(0.78-1.31)$ \\
\hline$F Y N$ & rs6910116 & 0.9928 & 0.9663 & $\mathrm{~T} / \mathrm{C}$ & $\mathrm{T}(14.9)$ & 6 & 112182621 & 0.9928 & $1.01(0.76-1.33)$ & $1.05(0.86-1.28)$ & $1.00(0.72-1.39)$ \\
\hline
\end{tabular}

Abbreviations: BH, Benjamini-Hochberg; Cl, confidence interval; db SNP, single-nucleotide polymorphism database; FDR, false discovery rate; MAF, minor allele frequency; OR, odds ratio. aRaw $P$-values were calculated in trend model.

b100000 permutations for single SNP association in trend model.

CMAF among controls.

dAdjusted FDR-BH (FDR estimated using the BH method) $P$-values calculated by the most significant SNPs of all genes in analysis. Bonferroni-adjusted $P$-values for multiple comparisons (number of tests $=22$ individual SNPs) were not significant $(P>0.4)$.

eCalculated in additive model with adjustment for gender and age.

${ }^{f}$ Conditional OR calculated among 290 patients and 290 controls matched for gender and age.

${ }^{*} P<0.05,{ }^{* *} 0.05 \leqslant P<0.10$

$P$-values $>0.05)$. The analysis of 290 age- and gender-matched sets showed that for the carriers of DCC rs17468382, CHP rs6492998 and RRAS2 rs2970332 SNPs, the ORs were 1.92, 0.77 and 0.79, respectively $(P<0.10)$. The hazard ratio of carrying DCC rs17468382 SNP was $1.80(P<0.05)$. The carriers of CHP rs6492998 and RRAS2 rs2970332 SNPs had reduced hazard ratios $(P<0.05)$.

The genetic risk score was analyzed for all 22 SNPs, and then for the set of four statistically significant SNPs in the DCC, CHP, RRAS2 and EPHB1 genes (Table 3 ). The mean genetic risk score was significantly higher for PD patients than for normal controls, regardless of the SNP combination. All genetic risk scores calculated using the number of risk alleles or risk estimates were significantly associated with PD risk. The risk estimates showed only a threefold increased risk $(2.95,95 \%$ CI (1.79-4.87) for the four significant SNPs; 2.84 , 95\% CI (1.82-4.42) for all of the SNPs). The genetic risk scores were similar for the subset of four SNPs (DCC, CHP, RRAS2 and EPHB1) and the complete set of 22 SNPs. The area under the receiver operating characteristic curve varied from 59.5 to $60.6 \%$. 
Table 3 Comparison of genetic risk score between total PD patients and normal controls

\begin{tabular}{lcccc}
\hline & Patients mean (s.d.) ${ }^{\text {a }}$ & Normal controls mean (s.d.) & OR (95\% Cl) & AUC value (95\% Cl) \\
\hline Genetic risk score & & & & \\
Based on significant SNPs ${ }^{\mathrm{b}}$ & $0.808(0.32)$ & $0.709(0.28)$ & $2.95(1.79-4.87)^{*}$ & $0.595(0.554-0.637)$ \\
Total SNPs on axon guidance pathwayd & $1.997(0.38)$ & $1.863(0.34)$ & $2.84(1.82-4.42)^{*}$ & $0.606(0.563-0.648)$ \\
\hline
\end{tabular}

Abbreviations: AUC, area under the receiver operating characteristic curve; $\mathrm{Cl}$, confidence interval; OR, odds ratio; SNP, single-nucleotide polymorphism; PD, Parkinson's disease;

Estimation of genetic score: risk estimates ( $\beta$-values calculated in the additive model) for each SNP were multiplied by 0,1 or 2 according to the number of risk alleles carried by each person.

aPatients diagnosed as PD.
${ }^{b}$ Calculated in additive model with adjustment for gender and age.

cFour SNPs of rs17468382 ( $\beta=0.7064)$, rs6492998 $(\beta=0.266)$, rs2970332 $(\beta=0.2111)$ and $\mathrm{rs} 2030737(\beta=0.256)$.

${ }^{\mathrm{d} A l l} 22$ SNPs of rs17468382 ( $\left.\beta=0.7064\right), \mathrm{rs} 6492998(\beta=0.266), \mathrm{rs} 2970332(\beta=0.2111), \mathrm{rs} 2030737(\beta=0.256), \mathrm{rs} 9688032(\beta=0.1459), \mathrm{rs} 2068435$ ( $\left.\beta=0.1569\right), \mathrm{rs} 12658266$

( $\beta=0.0894)$, rs739043 ( $\beta=0.0801)$, rs4444836 $(\beta=0.0818)$, rs11185076 ( $\beta=0.0587)$, rs $16830689(\beta=0.1171)$, rs1 $1097458(\beta=0.0620)$, rs153690 $(\beta=0.0348)$, rs17641276

$(\beta=0.0265), \operatorname{rs} 2044041$ ( $\beta=0.0154)$, rs6656034 ( $\beta=0.0184), \operatorname{rs} 2072952(\beta=0.0253), \mathrm{rs} 4678260$ ( $\beta=0.0237), \mathrm{rs} 10917325$ ( $\beta=0.00634), \mathrm{rs} 12740705$ ( $\beta=0.0197), \mathrm{rs} 6692804$

$(\beta=0.00841)$ and $\mathrm{rs} 6910116(\beta=0.0054)$.

${ }^{*} P<0.0001$

Table 4 Genes in axon guidance pathway and the risk of PD associated with selected combinations in MDR method

\begin{tabular}{|c|c|c|c|c|c|c|}
\hline & Test accuracy & CVC & P-value a & Patients & Controls & OR $(95 \% C l)^{\mathrm{b}}$ \\
\hline \multicolumn{7}{|l|}{ Total } \\
\hline CHP (rs6492998) & 0.5401 & $9 / 10$ & 0.1719 & $214(59.6)$ & $196(52.1)$ & 1.0 (reference) \\
\hline \multirow[t]{2}{*}{ GNAI3, FYN (rs6692804, rs6910116) } & 0.5684 & $6 / 10$ & 0.6230 & $157(42.9)$ & $213(56.2)$ & 1.0 (reference) \\
\hline & & & & $209(57.1)$ & $166(43.8)$ & $1.7(1.3-2.3)^{*}$ \\
\hline \multicolumn{7}{|l|}{ Matched } \\
\hline
\end{tabular}

Abbreviations: $\mathrm{CI}$, confidence interval; CVC, cross-validation consistency; OR, odds ratio.

a $P$-values were estimated by sign test.

bCalculated with adjustment for gender and age among total subjects.

${ }^{*} P<0.05$.
$P$

Table 4 summarizes the results of MDR and association analyses between the PD risk and combinations of high-risk genotypes suggested by the MDR analysis. Although all of the sign tests indicated the absence of significant MDR combinations, the global ORs showed significant results. The CHP rs6492998 SNP was present in the first list with a significant main effect both in all subjects and in age- and gender-matched subjects, and showed a protective effect for the PD risk (0.7, 95\% CI (0.6-1.0) for all subjects; 0.7, 95\% CI (0.5-1.0) for matched subjects).

There was no significant interaction between SNPs in logistic regression analysis using interaction terms of SNPs, but there was a marginally significant interaction between FYN rs6910116 and SLIT3 rs9688032 SNPs only among matched subjects (Table 5). However, both of these SNPs were not significant in the single marker analysis (Table 2). None of these results are consistent with the data reported by Lesnick et al. ${ }^{13}$

\section{DISCUSSION}

In our population, only 4 of the 22 SNPs that were reported to be significantly associated with $\mathrm{PD}$ risk by Lesnick et al. ${ }^{13}$ showed significant or marginally significant associations in analyses of single SNPs. SEMA5A gene was thought to have a role in the axon guidance of developing dopaminergic neurons and to interact with other proteins of the axon guidance pathway, ${ }^{10-12}$ thus leading to the idea of genetic variability in the axon guidance pathway as a possible susceptibility factor for PD.

The combination of the four risk genes was associated with only a threefold increased risk for PD, which suggest that the genes of the axon guidance pathway have only a minor role among Koreans. In particular, the PD risk as assessed by the genetic risk score and area under the curve for the 4 significant SNPs was similar to that for all 22 SNPs. This finding suggests that most of the influence from the entire set of 22 SNPs originates from the reported set of 4 SNPs. Lesnick et al. ${ }^{13}$ reported significant associations between PD risk and the SNPs in axon guidance pathway genes, with the effects being far greater for the pathway as a whole than for a single gene. However, we failed to replicate these findings in our Korean population. No significant interactions of the PD risk were found in the MDR analyses and logistic regression analyses using interaction terms. As it has been shown that PD risk alleles may greatly vary between populations, ${ }^{4-9,13-16}$ it is conceivable that the selected SNPs for this study are not the best candidates in Koreans because of the ethnicity variations. It may be that, owing to the different haplotype structure and allele frequencies in the Korean population, other SNPs may better tag the axonal guidance pathway and have a better chance for success. If genetic susceptibility factors are population specific, ${ }^{4-9,13-16}$ other SNPs or genes in the axonal guidance pathway might have a role in the PD pathogenesis in Koreans.

Our results suggest that in the Korean population, the 4 SNPs in the DCC, CHP, RRAS2 and EPHB1 genes among the selected set of 22 SNPs in genes of the axon guidance pathway be regulating factors associated with PD development; however, their influence is minor. No interactions were found among the SNPs, suggesting that in contrast to the data reported by Lesnick et al., ${ }^{13}$ the effect for the pathway as a whole is not greater than that for single genes in the Korean population. Further studies involving populations of various ethnicities and replication studies focused on epistasis are needed to elucidate the ethnic specificity of the genes of the axon guidance pathway. 
Table 5 Interaction analysis suggested by Lesnick et al.

\begin{tabular}{|c|c|c|c|}
\hline Gene & $d b S N P I D$ & OR $(95 \% C l)^{\mathrm{a}}$ & OR $(95 \% \mathrm{Cl})^{\mathrm{b}}$ \\
\hline DCC, PAK4 & rs17468382, rs17641276 & $1.38(0.36-2.40)$ & $1.35(0.24-2.45)$ \\
\hline EPHA4, FYN & rs13386128, rs6910116 & $-^{c}$ & $-^{c}$ \\
\hline EPHB2, EFNA5 & rs10917325, rs153690 & $0.94(0.63-1.24)$ & $0.98(0.64-1.32)$ \\
\hline FYN, RRAS2 & rs6910116, rs2970332 & $0.73(0.31-1.16)$ & $1.78(0.30-1.25)$ \\
\hline FYN, SLIT3 & rs6910116, rs9688032 & $1.29(0.88-1.69)$ & $1.59(1.12-2.07)^{*}$ \\
\hline SEMA5A, RAC2 & rs12658266, rs739043 & $1.21(0.88-1.62)$ & $1.32(0.95-1.70)$ \\
\hline UNC5C1, DCC & rs11097458, rs17468382 & $0.71(0.20-1.62)$ & $0.54(0.25-1.58)$ \\
\hline
\end{tabular}

Abbreviations: $\mathrm{Cl}$, confidence interval; db SNP, single-nucleotide polymorphism database; OR, odds ratio.

${ }^{a}$ Calculated among total subjects with adjustment for gender and age.

bCalculated among 290 patients and 290 controls matched for gender and age.

CEPHA4 was excluded because of minor allele frequency $<0.01$ among study population.

${ }^{*} P<0.05$.

\section{CONFLICT OF INTEREST}

Eun-Soon Shin is employed by a commercial company, DNA Link (http://www.dnalink.com). All of the authors confirm that this employment does not alter the adherence to all the Journal of Human Genetics policies on sharing data and materials and preparing the paper.

\section{ACKNOWLEDGEMENTS}

This study was supported in part by a grant from the Korean Health $21 \mathrm{R}$ and D Project, Ministry of Health and Welfare, Republic of Korea (A030001) and a research grant from the Seoul National University Bundang Hospital (02-2009017). We acknowledge a generous donation from Mr Chung Suk-Gyoo and Sinyang Cultural Foundation. We thank Dr Paul Kolston from English Science Editing for the assistance with preparation of the manuscript. With the exception of this funder, the other funders had no role in study design, data collection and analysis, decision to publish or preparation of the paper.

1 Lees, A. J., Hardy, J. \& Revesz, T. Parkinson's disease. Lancet 373, 2055-2066 (2009).

2 Klein, C. \& Schlossmacher, M. G. Parkinson disease, 10 years after its genetic revolution: multiple clues to a complex disorder. Neurology 69, 2093-2104 (2007).

3 Yang, Y. X., Wood, N. W. \& Latchman, D. S. Molecular basis of Parkinson's disease. Neuroreport 20, 150-156 (2009).

4 Maraganore, D. M., de Andrade, M., Lesnick, T., Strain, K. J., Farrer, M. J., Rocca, W. A. et al. High-resolution whole-genome association study of Parkinson disease. Am. J. Hum. Genet. 77, 685-693 (2005).

5 Fung, H. C., Scholz, S., Matarin, M., Simon-Sanchez, J., Hernandez, D., Britton, A. et al. Genome-wide genotyping in Parkinson's disease and neurologically normal controls: first stage analysis and public release of data. Lancet Neurol. 5, 911-916 (2006).

6 Pankratz, N., Wilk, J. B., Latourelle, J. C., DeStefano, A. L., Halter, C., Pugh, E. W. et al. Genomewide association study for susceptibility genes contributing to familial Parkinson disease. Hum. Genet. 124, 593-605 (2009).

7 Satake, W., Nakabayashi, Y., Mizuta, I., Hirota, Y., Ito, C., Kubo, M. et al. Genome-wide association study identifies common variants at four loci as genetic risk factors for Parkinson's disease. Nat. Genet. 41, 1303-1307 (2009).

8 Simón-Sánchez, J., Schulte, C., Bras, J. M., Sharma, M., Gibbs, J. R., Berg, D. et al. Genome-wide association study reveals genetic risk underlying Parkinson's disease. Nat. Genet. 41, 1308-1312 (2009).

9 Edwards, T. L., Scott, W. K., Almonte, C., Burt, A., Powell, E. H., Beecham, G. W. et al. Genome-wide association study confirms SNPS in SNCA and the MAPT region as common risk factors for Parkinson disease. Ann. Hum. Genet. 74, 97-109 (2010).
10 Kawano, H., Horie, M., Honma, S., Kawamura, K., Takeuchi, K. \& Kimura, S. Aberrant trajectory of ascending dopaminergic pathway in mice lacking Nkx2.1. Exp. Neurol. 182, 103-112 (2003).

11 Chilton, J. K. Molecular mechanisms of axon guidance. Dev. Biol. 292, 13-24 (2006).

12 Gomez, T. M. \& Zheng, J. Q. The molecular basis for calcium-dependent axon path finding. Nat. Rev. Neurosci. 7, 115-125 (2006).

13 Lesnick, T. G., Papapetropoulos, S., Mash, D. C., Ffrench-Mullen, J., Shehadeh, L., de Andrade, M. et al. A genomic pathway approach to a complex disease: axon guidance and Parkinson disease. PLoS Genet. 3, e98 (2007).

14 Srinivasan, B. S., Doostzadeh, J., Absalan, F., Mohandessi, S., Jalili, R., Bigdeli, S. et al. Whole genome survey of coding SNPs reveals a reproducible pathway determinant of Parkinson disease. Hum. Mutat. 30, 228-238 (2009).

$15 \mathrm{Li}$, Y., Rowland, C., Xiromerisiou, G., Lagier, R. J., Schrodi, S. J., Dradiotis, E. et al. Neither replication nor simulation supports a role for the axon guidance pathway in the genetics of Parkinson's disease. PLoS One 3, e2707 (2008).

16 Yun, J. Y., Lee, W. W., Lee, J. Y., Kim, H. J., Park, S. S. \& Jeon, B. S. SNCA variants associated with increased risk of multiple system atrophy. Ann. Neurol. 67, 554-555 (2010).

17 Hughes, A. J., Daniel, S. E., Kilford, L. \& Lees, A. J. Accuracy of clinical diagnosis of idiopathic Parkinson's disease: a clinico-pathological study of 100 cases. J. Neurol. Neurosurg. Psychiatry 55, 181-184 (1992).

18 Kim, J. M., Hong, S., Kim, G. P., Choi, Y. J., Kim, Y. K., Park, S. S. et al. Importance of low-range CAG expansion and CAA interruption in SCA2 Parkinsonism. Arch. Neurol. 64, 1510-1518 (2007).

19 Cho, J. W., Kim, S. Y., Park, S. S., Kim, H. J., Ahn, T. B., Kim, J. M. et al. The G2019S LRRK2 mutation is rare in Korean patients with Parkinson's disease. Can. J. Neurol. Sci. 34, 53-55 (2007).

20 Ahn, T. B., Kim, S. Y., Kim, J. Y., Park, S. S., Lee, D. S., Min, H. J. et al. $\alpha$-Synuclein gene duplication is present in sporadic Parkinson disease. Neurology 70, 43-49 (2008).

21 Kim, J. Y., Kim, S. Y., Kim, J. M., Kim, Y. K., Yoon, K. Y., Kim, J. Y. et al. Spinocerebellar ataxia type 17 mutation as a causative and susceptibility gene in parkinsonism. Neurology 72, 1385-1389 (2009).

22 Cho, J. W., Kim, S. Y., Park, S. S. \& Jeon, B. S. The G2019S LRRK2 mutation is rare in Korean patients with Parkinson's disease and multiple system atrophy. J. Clin. Neurol. 5, 29-32 (2009).

23 Benjamini, Y. \& Hochberg, Y. Controlling the false discovery rate: a practical and powerful approach to multiple testing. J. Roy. Stat. Soc. B. 57, 289-300 (1995)

24 Talmud, P. J., Hingorani, A. D., Cooper, J. A., Marmot, M. G., Brunner, E. J., Kumari, M. et al. Utility of genetic and non-genetic risk factors in prediction of type 2 diabetes: whitehall II prospective cohort study. BMJ 340, b4838 (2010).

25 Hahn, L. W., Ritchie, M. D. \& Moore, J. H. Multifactor dimensionality reduction software for detecting gene-gene and gene-environment interactions. Bioinformatics 19, 376-382 (2003).

26 Moore, J. H., Gilbert, J. C., Tsai, C. T., Chiang, F. T., Holden, T., Barney, N. et al. A flexible computational framework for detecting, characterizing, and interpreting statistical patterns of epistasis in genetic studies of human disease susceptibility. J. Theor. Biol. 241, 252-261 (2006).

27 Purcell, S., Neale, B., Todd-Brown, K., Thomas, L., Ferreira, M. A., Bender, D. et al. PLINK: a tool set for whole-genome association and population-based linkage analyses. Am. J. Hum. Genet. 81, 559-575 (2007).

Supplementary Information accompanies the paper on Journal of Human Genetics website (http://www.nature.com/jhg) 